\title{
SHP2 Inhibitor RMC-4630
}

National Cancer Institute

\section{Source}

National Cancer Institute. SHP2 Inhibitor RMC-4630. NCI Thesaurus. Code C155850.

An orally bioavailable inhibitor of protein tyrosine phosphatase (PTP) non-receptor type

11 (SHP2; Src homology region 2 domain phosphatase; PTPN11), with potential antineoplastic activity. Upon oral administration, SHP2 inhibitor RMC-4630 targets, binds to and inhibits the activity of SHP2. This prevents SHP2-mediated signaling, inhibits MAPK signaling and prevents growth of SHP2-expressing tumor cells. SHP2, an oncoprotein overexpressed in a variety of cancer cell types, regulates cell survival, differentiation and proliferation through activation of the RAS-RAF-MEK-ERK signaling pathway. The RAS-MAPK pathway is often hyperactivated in cancer cells due to specific mutations and rearrangements and are dependent on SHP2 for their oncogenic signaling. SHP2 also regulates programmed cell death 1 (PD-1)-mediated signal transduction and is involved in immune checkpoint modulation. 